\title{
Improving the accuracy and turn-around time of controlled drug prescribing for patients being discharged home for end-of-life care
}

\author{
Suzanne Daniel, Janki Patel, Debbie Benson \\ Conquest Hospital, East Sussex Healthcare NHS Trust, England
}

\begin{abstract}
Many patients approaching the end of their life express the preference to die at home,[1] although unfortunately the majority of people will still die in hospital.[2] For patients approaching the end of their life, it was noted anecdotally that often those who have expressed a preference to go home from hospital for end of life care may have their discharge delayed due to problems in the prescribing of common medications used to alleviate distressing symptoms at the end of life.

An initial audit at Conquest Hospital showed an $89 \%$ error rate in these prescriptions, mostly related to prescribing controlled drugs such as morphine and midazolam. A single standardised dispensing chart for commonly prescribed medications at the end of life, in the form of both "Just in Case" medications and syringe driver medications, was created which addressed this problem by having the medications pre-written so as to meet all legal requirements for controlled drugs. The prescriber is able to choose and fill out an appropriate drug and dose by using flow-chart information overleaf and then sign the prescription to allow it to be dispensed. After an initial two month pilot period, a re-audit showed a significant fall in error rate down to $11 \%$, as well as an improvement in turnaround time in dispensing the medications.
\end{abstract}

\section{Problem}

In the last few days of life, a patient may no longer be able to swallow their oral medications and may require parenteral medications to manage symptoms such as pain, nausea and vomiting, respiratory secretions, and agitation. These symptoms can often be anticipated, and if a patient has expressed a preference to die at home and is leaving hospital then anticipatory injectable medication, or "Just in Case" medications, can be dispensed at discharge for administration in by appropriately trained community nurses.

Some of these medications will be controlled drugs. The current process for the prescription of these drugs for palliative patients that are waiting discharge home from East Sussex Healthcare NHS Trust (ESHT), England, is open to a number of sources of error, and these have knock-on effects against achieving a safe and quick discharge for these patients. While all other medications can be ordered electronically via the medicines management system, a prescription for a controlled drug must be dispensed from a drug chart. If an incorrectly written controlled drug prescription is sent to pharmacy, the pharmacist will contact the prescribing doctor and make arrangements for amendments to be made. This may be via transferring the drug chart back to the ward or the doctor coming to pharmacy to amend it, or the pharmacist trying to find the doctor themselves to make the relavent amendments.

As these discharge medications are written onto the patients usual inpatient drug chart, sending the chart to the pharmacy department means that the patient is on the ward without their drug chart while the prescription error is corrected or is being dispensed.

Consequently the patient is unable to have any of their regular or "as required" medications during that time.
Additionally, there is a lack of standardisation within ESHT as to the choice, doses and the quantity to be supplied of these agents. It is recognised that there needs to be flexib

ility for individual patients, especially in the prescribing of opiates or in the event of renal impairment. However, guidelines for several of the "Just in Case" medications would be useful for those patients who are being discharged for terminal care in the community.

\section{Background}

End of life care refers to the management of patients during their last few days, weeks, or months of life. Although $63 \%$ of people express a preference to die in their own home,[1] only $19 \%$ of people will do so, and $58 \%$ of deaths in England occur in hospital.[2]

The NICE quality standard for end of life care for adults sets out markers of high quality care for adults aged 18 years and older with advanced, progressive, incurable conditions. It aims to contribute to the following outcomes for people approaching the end of life:

- The care that people approaching the end of life receive is aligned to their needs and preferences

- Increased length of time spent in preferred place of care during the last year of life

- Reduction in unscheduled care hospital admissions leading to death in hospital (where death in hospital is against their stated preference).[3]

Some of the "Just in Case" medications that may be used to gain symptom control for a patient as they are deteriorate, and frequently the drugs used in syringe drivers, will be controlled drugs that have 
tighter regulations regarding their use, storage, and prescription requirements. A prescription for a controlled drug has important differences as to how it is written compared to regular prescriptions, and legally must contain the following details:

- The patient's full name, address, and age (if under 12 years old)

- The name and form of the drug, even if only one form exists

- The strength of the preparation

- Specific instructions on the dose to be taken

- The total quantity (in words and figures) or total number of dosage units (in words and figures) of the drug to be supplied.[4]

\section{Baseline measurement}

In conjunction with the palliative care team and pharmacy department, over a two week period we identified all patients going home from Conquest Hospital for end of life care with a "Just in Case" prescription. We developed an audit proforma for use within the pharmacy department and measured the drug and doses prescribed, any sources of error, and the time taken from the prescription arriving in the pharmacy department to medications being released to the ward.

Nine patients were discharged home from hospital for end-of-life care during the two week audit period with prescriptions for 'Just in Case' medications. Eight of the nine prescriptions (89\%) contained at least one prescription error which required amending prior to the prescription being dispensed by the pharmacy department. The average turnaround time for a prescription to be dispensed was 1 hour and 25 minutes.

The most common prescription error $(67 \%)$ was missing information regarding the strength of the drug being prescribed. The legal requirement to write the total quantity of a controlled drug to be supplied in both words and figures was only written in $44 \%$ of prescriptions. Other errors noted were the frequency of dosing missed (33\%), patient details missing (22\%), and prescriber signature missing $(11 \%)$

\section{Design}

To rectify these problems we developed a standardised dispensing chart for commonly prescribed medications at the end of life (see appendix 1). The chart allows a single form to be completed for either (or both) "Just in Case" medications and syringe driver medication, should the patient require them. The chart is written clearly with the names of the first-choice drugs to treat pain, nausea and vomiting, anxiety, confusion or restlessness, and respiratory secretions, with spaces for the prescribing doctor to fill out the dose required and sign the prescription. Overleaf, flow-diagram information is provided on how to pick a suitable dose. Controlled drugs are written so as to meet all legal prescribing requirements providing that the prescriber fills in all the blanks.
The dispensing chart is easily recognisable and is given priority through the pharmacy department to allow a more rapid dispensing process. The creation of a separate dispensing chart for end of life medications also allows the patient's regular inpatient chart to stay with them on the ward while their discharge medications are being prepared, meaning they can receive they regular medications and any "as required" medications during this time.

\section{Strategy}

A 'Plan Do Study Act' approach was implemented and the chart was amended following feedback from users. The majority of changes related to small administrative improvements, for example by providing spaces for the patient's ward and consultant, and for pharmacy staff to sign at the various dispensing stages. The recommended dose of haloperidol was also changed following consultation with the palliative care consultant.

See supplementary file: ds3826.pdf - "End of life drug dispensing chart"

\section{Post-measurement}

After a two month pilot period, we re-audited using the same audit proforma that had been implemented during our baseline measurements. During the fourteen days of data collection there were nine prescriptions received by the pharmacy department on the new standardised dispensing chart.

Only one of these prescriptions (11\%) had an error requiring correction, which was where the prescribing doctor had not filled out the gap to detail the strength of diamorphine to be dispensed. The average time for dispensing the medications was 60 minutes.

All prescriptions received met legal requirements for controlled drug prescribing in terms of having the full patient details and total quantity supplied in both words and figures.

\section{Lessons and limitations}

A major limitation of this project was the small sample size of the audits, which reflects that there are only a reasonably small number of people who go home from hospital for end of life care. People may alternatively decide that they wish to die in hospital or at a hospice, or unfortunately may not have the opportunity or capacity to express their preference so consequently would not benefit from our drug dispensing chart.

The frequent turnover of house officers and senior house officers, who were identified in our audit as the most frequent prescribers for end of life medications, also means that staff training on the dispensing charts has to be ongoing. To address this, sessions on the charts and controlled drug prescribing in general have been added to the compulsory local F1 and F2 teaching schedule.

\section{Conclusion}




\section{BMJ Quality Improvement Reports}

Despite there being a relatively small number of people who do go home from hospital for end of life care, any intervention that makes this process smoother and safer is valuable. Anecdotal observations had been made that the process of dispensing medications for these patients was often delayed due to errors in the prescription of controlled drugs, and an initial audit supported this view by identifying that $89 \%$ of end-of-life prescriptions contained at least one error. The majority of these errors related to meeting the legal requirements for the prescription of controlled drugs.

To rectify this, we created a standardised drug dispensing chart for end of life medications which met all legal requirements for controlled drugs prescriptions as well as providing easy to follow guidance on drug choice and dose to be started on. Re-audit after a pilot period showed a significant improvement in both error rate and dispensing time of these medications. This then allowed for better use of staff time within the pharmacy department, and a smoother and safer discharge for our patients going home for end of life care.

\section{References}

1. National End of Life Care Intelligence Network. Variations in place of death in England. Bristol: National End of Life Care Intelligence Network; 2010.

2. Gomes B, Calanzani N, Higginson I. Local preferences and place of death in regions within England 2010. London: Cicely Saunders International; 2011.

3. National Institute for Health and Care Excellence. Quality standard for end of life care for adults. Aug 2011 (modified October 2013). London: National Institute for Health and Clinical Excellence; 2013.

4. Joint Formulary Committee. British National Formulary 66. London: BMJ Group \& Pharmaceutical Press; 2013.

\section{Declaration of interests}

None.

\section{Acknowledgements}

Dr Elena Mucci, ESHT acute specialist palliative care nurses (Conquest site). 